\title{
DRESS Syndrome- Uncommon Drug Reaction with Common Disease Treatment: A Case Report
}

\author{
B Saroj Kumar Prusty ${ }^{1}$, Majed Abdulbasit Momin ${ }^{2}$, Yugvaveer K Goud ${ }^{3}$, Kiran Kumar Ramineni ${ }^{4}$, Safina \\ Perveen $^{1}$ \\ ${ }^{1}$ Yashoda Hospitals, Malakpet Branch Hyderabad, Department of Critical Medicine, Hyderabad, India \\ ${ }^{2}$ Yashoda Hospitals, Malakpet Branch Hyderabad, Department of Laboratory Medicine, Hyderabad, India \\ ${ }^{3}$ Yashoda Hospitals, Malakpet Branch Hyderabad, Department of Pulmonology, Hyderabad, India \\ ${ }^{4}$ Yashoda Hospitals, Malakpet Branch Hyderabad, Department of Neurology, Hyderabad, India
}

\begin{abstract}
Drug Reaction with Eosinophilia and Systemic symptoms (DRESS syndrome), is a severe adverse reaction associated with diverse collection of drugs, characterized by severe mucocutaneous rash, eosinophilia, fever, lymphadenopathy and extensive systemic involvement. We report a case of a 21 -year-old female who developed clinical manifestations of fever, maculopapular rash, lymphadenopathy, eosinophilia and systemic symptoms after taking antitubercular medication for pulmonary tuberculosis, with subsequent development of acute liver failure with encephalopathy and coagulopathy. She was managed successfully with withdrawal of the offending medication and supportive care in intensive care unit. This case highlights the importance of consideration of antitubercular medication related drug reaction even with delayed onset of symptoms. J Microbiol Infect Dis 2020; 10(4): $225-229$.
\end{abstract}

Keywords: Anti-tubercular treatment, DRESS syndrome, Eosinophilia, Management

\section{INTRODUCTION}

Drug Reaction with Eosinophilia and Systemic symptoms (DRESS) syndrome is a rare, delayed cutaneous hypersensitivity reaction, seen more commonly with anticonvulsants, sulfonamides, anti-depressants, anti-inflammatory agents, antivirals, ACE inhibitors and Beta blockers etc. [1]. Although tuberculosis is a common infectious disease in developing countries, like India, drug reaction with Antitubercular therapy (ATT) has not been routinely reported as the causative agent for DRESS syndrome.

This syndrome causes a diverse array of clinical symptoms, usually two to eight weeks after initiating the offending drug. The clinical triad of DRESS syndrome consists of fever, skin rash and internal organ involvement. Delayed onset of these symptoms is an important feature of DRESS syndrome and clinical outcome also takes a long time after withdrawal of the culprit drug [2]. It is difficult to diagnose, as many of its clinical features mimic those found with other serious systemic disorders. However, early recognition of the syndrome with cessation of the causative drug is essential in improving the outcome of this potentially fatal condition.

\section{CASE REPORT}

A 21-year-old female was brought to the emergency department with complaints of fever, vomiting, rash for 10 days and facial puffiness yellowish discoloration of eyes for seven days. Fever was of high grade, intermittent and associated with chills. Erythematous maculopapular rash was present all over the body including face and was associated with itching. She later developed facial puffiness and noticed yellowish discoloration of both eyes. She was diagnosed with pulmonary tuberculosis 6 weeks back and was started on Anti Tubercular drugs (Ethambutol $800 \mathrm{mg}$, Isoniazid $300 \mathrm{mg}$, Pyrazinamide $1500 \mathrm{mg}$ and Rifampicin $450 \mathrm{mg}$ ).

General examination revealed icterus, facial puffiness and erythematous, morbilliform and pruritic rash over face, arms, trunk and legs with intact mucous membranes.

Correspondence: Dr. Majed Abdulbasit Momin, Yashoda Hospitals, Malakpet Branch Hyderabad, Department of Laboratory Medicine, Hyderabad, India

Email: majedmomin9@gmail.com

Received: 03 September 2019 Accepted: 25 July 2020

Copyright (C JMID / Journal of Microbiology and Infectious Diseases 2020, All rights reserved 


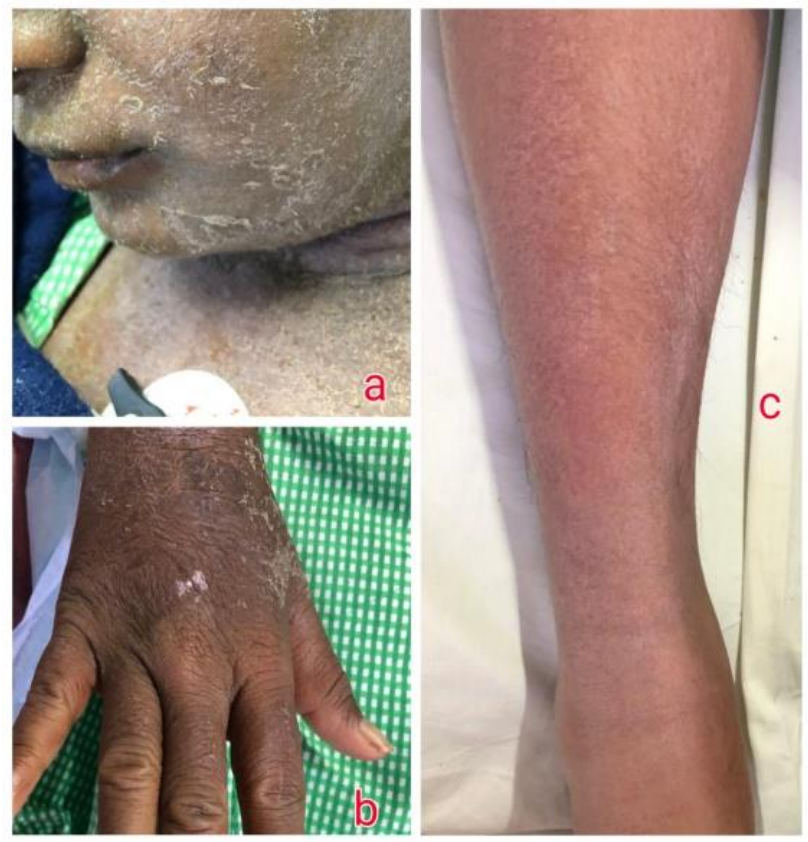

Figure 1. Diffuse Erythematous morbilliform, and exfoliative rashes all over the body including face, legs, upper limbs and chest.

Three posterior auricular lymph nodes were palpable on right side, which were of around $2 \mathrm{~cm}$ size, discrete and soft in consistency. Abdominal palpation revealed right hypochondrial tenderness and mild splenomegaly. Ultrasonography of abdomen and pelvis showed hepatomegaly $(174 \mathrm{~mm})$, splenomegaly $(141 \mathrm{~mm})$, gallbladder wall edema and minimal free fluid in the right pleural cavity.

The lab investigation showed raised bilirubin, SGOT, and SGPT (features of acute hepatitis), prolonged PT/INR, APTT (coagulopathy), and leukocytosis with significant peripheral eosinophilia. Based on the typical clinical picture with supportive laboratory parameters a provisional diagnosis of DRESS Syndrome was made. Serial Laboratory results were abnormal as mentioned in the (Table1)

Ultrasound guided FNAC of cervical lymph node was suggestive of reactive lymphadenitis. Serology work up for other infective etiologies was negative including HIV 1 \& 2, Hepatitis A, B, C\& E, Dengue and EBV. Influenza Screening including $\mathrm{H} 1 \mathrm{~N} 1$ was negative.

She was admitted in intensive care unit and antitubercular drugs were stopped. Within 48 hours of admission patient landed in acute liver failure with worsening of coagulopathy and hepatic encephalopathy of grade 2. She had recurrent hypoglycemic episodes secondary to acute liver failure. In addition to supportive care she was managed with injection N-Acetyl Cystein IV 100 $\mathrm{mg} / \mathrm{kg} /$ day as intravenous infusion for seven days, injection vitamin $\mathrm{K} 10 \mathrm{mg}$ intravenous once daily for initial 5 days, intravenous hydrocortisone $100 \mathrm{mg}$ thrice daily for one week followed by oral prednisolone $1 \mathrm{mg} / \mathrm{kg}$ for two weeks which was gradually tapered over next six weeks till complete resolution of eosinophilia and skin rashes. As she had worsening liver enzymes and encephalopathy on day 5, plasmapheresis was started with $3000 \mathrm{ml}$ of fresh frozen plasma for consecutive three days. Patient's general condition, neurological status, skin rash and laboratory parameters including Absolute eosinophilic count, liver enzymes, and PT with INR improved significantly with plasmapheresis and other supportive measures. She was successfully discharged on day 27 in a stable condition. Modified ATT including Levofloxacin $750 \mathrm{mg}$ once daily, Amikacin 1gm IV once daily and Ethambutol $800 \mathrm{mg}$ once daily were initiated. On follow up at 6 weeks, 8 weeks and 12 weeks she maintained clinical and laboratory parameter improvement and was independent for activities of daily living. So modified ATT as prescribed above was continued for 2 months and amikacin was stopped. Isoniazid and cycloserine were added sequentially without any adverse effects.

\section{DISCUSSION}

The term DRESS syndrome was first coined by Bocquet et al in 1996. As per Bocquet's criteria the diagnosis of DRESS syndrome requires the following 3 features: 1) skin eruption, 2) blood eosinophilia $(>1.5 \times 103 / \mu \mathrm{L})$ or the presence of atypical lymphocytes, and 3 ) internal organ involvement, including lymphadenopathies (>2 $\mathrm{cm}$ in diameter), hepatitis (liver transaminases values $>$ twice the upper normal limit), interstitial nephritis, and interstitial pneumonia or carditis [3]. Fever and rash are the common initial symptoms. Rash is usually macular, appears first on the face, abdomen and upper limbs. Lymphadenopathy is seen in $75 \%$ cases and liver is the most commonly affected internal organ. 
Table-1. Laboratory parameters of the patient.

\begin{tabular}{|c|c|c|c|c|c|c|c|}
\hline Variables & Day 1 & Day 3 & Day 5 & Day 10 & Day 15 & Day 20 & Day 27 \\
\hline Hemoglobin gm\% & 111.6 & 10.5 & 10 & 7.1 & 8.5 & 8.6 & 9.1 \\
\hline TLC cells/cumm & 37100 & 40800 & 28900 & 15400 & 15200 & 14100 & 13600 \\
\hline $\begin{array}{l}\mathrm{DC} \% \mathrm{~N}, \mathrm{~L}, \mathrm{E}, \mathrm{M}, \\
\mathrm{B}\end{array}$ & $3,20,22,5,0$ & $50,25,20,5,0$ & $52,20,24,4,0$ & $54,26,10,6,0$ & $59,22,11,4,0$ & $59,20,10,6,0$ & $59,22,9,5,0$ \\
\hline AEC cells/cmm & 8162 & 8160 & 6936 & 1540 & 1672 & 1410 & 1224 \\
\hline $\begin{array}{l}\text { Platelets } \\
\text { lacs/cumm }\end{array}$ & 3.62 & 2.50 & 1.70 & 1.80 & 1.40 & 2.10 & 2.20 \\
\hline T. bilirubin mg/dl & 5 & 7.3 & 10.5 & 9.44 & 10.25 & 16.27 & 5.2 \\
\hline SGOT U/L & 450 & 465 & 547 & 94 & 85 & 112 & 53 \\
\hline SGPT U/L & 232 & 330 & 405 & 170 & 56 & 55 & 20 \\
\hline ALP U/L & 277 & 236 & 159 & 99 & 91 & 161 & 166 \\
\hline GGT U/L & 123 & 110 & 56 & 60 & 69 & 110 & 182 \\
\hline PT secs/INR & j3.1/5.86 & $44.3 / 4.67$ & $39.5 / 4.05$ & 22.7/1.95 & $17.5 / 1.41$ & $18.9 / 1.55$ & $16 / 1.24$ \\
\hline aPTT secs & 70 & 72 & 60.8 & 36.4 & 36.2 & 34.6 & 32.1 \\
\hline RBS mg/dl & 92 & 65 & 72 & 75 & 70 & 80 & 100 \\
\hline $\begin{array}{l}\text { Serum Creatinine } \\
\mathrm{mg} / \mathrm{dl}\end{array}$ & 0.5 & 0.6 & 0.5 & 0.3 & 0.4 & 0.5 & 0.6 \\
\hline $\begin{array}{l}\mathrm{Na}, \mathrm{K}, \mathrm{Cl} \text { (Serum) } \\
\mathrm{meq} / \mathrm{L}\end{array}$ & $30 / 4.1 / 99$ & $136 / 3.9 / 99$ & 138/3.0/93 & $141 / 4.2 / 98$ & $136 / 3.4 / 96$ & $138 / 4.1 / 98$ & $140 / 3.8 / 96$ \\
\hline
\end{tabular}

Table 2: Drug groups associated with DRESS syndrome.

\begin{tabular}{|c|c|}
\hline Drug groups & Specific drug \\
\hline Anticonvulsants & Phenytoin, carbamazepine, phenobarbital, lamotrigine, valproate \\
\hline Anti-inflammatories & Piroxicam, naproxen, diclofenac, sulindac, ibuprofen, celecoxib \\
\hline Anti-infective agents & $\begin{array}{l}\text { Abacavir, telaprevir, nevirapine, linezolid, doxycycline, nitrofurantoin, } \\
\text { vancomycin, minocycline, ampicillin, isoniazid, rifampicin }\end{array}$ \\
\hline Antidepressants & Despiramine, amitriptyline, fluoxetine \\
\hline Sulfonamides/sulfones & Dapsone, sulfasalazine, trimethoptim-sulfametoxazole \\
\hline $\begin{array}{l}\text { Angiotensin-converting enzyme } \\
\text { inhibitors }\end{array}$ & Captopril, enalapril \\
\hline Beta-blockers & Atenolol, celiprolol \\
\hline
\end{tabular}

In 2007, Kardaun et al [4] of severe cutaneous adverse reaction (RegiSCAR) study group published a scoring system including various parameters like skin eruption, fever $\left(>38.5{ }^{\circ} \mathrm{C}\right)$, lymphadenopathy, involvement of at least 1 internal organ, blood eosinophilia $(>10 \%$ or $700 / \mu \mathrm{L}$ ), presence of atypical lymphocytes and negative evaluation for other potential causes. A composite score of more than five indicates a definite case of DRESS. Our case satisfied the criteria for the definite diagnosis of DRESS syndrome.

Although exact pathogenesis is not clear, both genetic and acquired factors related to drug metabolism contribute to the susceptibility for the development of DRESS in a given individual 
[5]. Various groups of drugs associated with DRESS include anticonvulsants, antibiotics, antivirals, analgesics etc. [6,7]. Details are summarized in Table 2.

DRESS syndrome usually develops within 2 months of ingestion of the offending drug. In addition to meticulous clinical examination, minimum evaluation required for diagnosis include hemogram with absolute eosinophil count, lymphocyte count, Liver function tests, serum creatinine, serum electrolytes, Electrocardiogram, Chest $X$ ray and ultrasound abdomen. Diagnostic differentials include Stevens-Johnson syndrome (SJS), Toxic epidermal necrolysis (TEN), hyper eosinophilic syndrome and Erythroderma [6].

The management protocols are not based on any consensus or guidelines. The primary strategy is to stop the offending drug. Systemic steroid therapy should begin with a minimum dose of $1.0 \mathrm{mg} / \mathrm{kg} / \mathrm{day}$ of prednisone or equivalent along with supportive care and close monitoring of clinical, laboratory parameters. Steroids need to be tapered slowly over 6-8 weeks, even upon clinical resolution, to prevent relapse [8]. Additional treatment options include intravenous immunoglobulins, rituximab and plasmapheresis [9].

In tuberculosis endemic countries like India, antituberculosis drugs are extensively prescribed sometimes even empirically. First line drugs including Rifampicin, pyrazinamide, isoniazid and streptomycin have been reported to cause DRESS syndrome [10]. It is challenging to identify ATT drugs as potential DRESS syndrome inducers, take the risk of stopping or modifying the standard drug regimen especially with the addition of corticosteroids in the context of active infection.

Approximately $10 \%$ mortality rate is seen in DRESS syndrome, mostly due to liver damage thought to be secondary to eosinophilic infiltration. The prognosis of patients with DRESS syndrome related acute liver failure without transplantation is very poor [11].

To conclude, DRESS syndrome is an underrecognized and potentially life-threatening hypersensitivity reaction. It is an extremely uncommon complication of antitubercular medication which is commonly prescribed even empirically in tuberculosis endemic zones like India. Coupled with meticulous clinical examination, judicious utilization of laboratory services helps in timely diagnosis, appropriate management and better outcome. Treating clinician should be vigilant and counsel the patients regarding this complication especially while prescribing the drugs associated with this syndrome.

\section{ACKNOWLEDGMENTS}

Conflict of interest: The authors declare no personal or financial conflict of interest.

Financial Disclosure: No financial support was received.

\section{REFERENCES}

1. Cacoub $P$, Musette $P$, Descamps $V$, et al. The DRESS syndrome: a literature review. Am J Med 2011; 124: 588-97.

2. Tas S, Simonart T. Drug rash with eosinophilia and systemic symptoms (DRESS syndrome). Acta Clin Belg 1999; 54:197-200.

3. Bocquet H, Bagot M, Roujeau JC. Drug-induced pseudolymphoma and drug hypersensitivity syndrome (Drug Rash with Eosinophilia and Systemic Symptoms: DRESS). Semin Cutan Med Surg 1996; 15: $250-7$

4. Kardaun SH, Sidoroff A, Valeyrie-Allanore L, et al. Variability in the clinical pattern of cutaneous sideeffects of drugs with systemic symptoms: does a DRESS syndrome really exist? Br J Dermatol 2007; 156:609-11.

5. Walsh SA, Creamer D. Drug reaction with eosinophilia and systemic symptoms (DRESS): a clinical update and review of current thinking. Clin Exp Dermatol 2011; 36:6-11.

6. De A, Rajagopalan M, Sarda A, Das S, Biswas P. Drug reaction with eosinophilia and systemic symptoms: An update and review of recent literature. Indian J Dermatol 2018; 63:30-40

7. Kömür S, Ulu A, Kurtaran B, İnal AS, Taşova $Y$, Aksu HS. Telaprevir-related DRESS syndrome complicating hepatitis $\mathrm{C}$ treatment. J Microbio Infect Dis 2015; 5(01):36-7.

8. Sultan SJ, Sameem F, Ashraf M. Drug reaction with eosinophilia and systemic symptoms: manifestations, treatment, and outcome in 17 patients. Int J Dermatol 2015; 54(5):537-42.

9. Shaughnessy K, Bouchard S, Mohr M, Herre J, Salkey K. Minocycline-induced drug reaction with eosinophilia and systemic symptoms (DRESS) syndrome with persistent myocarditis. J Am Acad Dermatol 2010; 62: 315-318. 
10. Palmero D, Castagnino J, Musella RM, Mosca C, González Montaner P, de Casado GC. Difficult clinical management of anti-tuberculosis DRESS syndrome. Int J Tuberc Lung Dis 2013; 17:76-8.

11. Ichai P, Laurent-Bellue A, Saliba F, et al. Acute liver failure/injury related to drug reaction with eosinophilia and systemic symptoms: outcomes and prognostic factors. Transplantation 2017; 101(8): 1830-7. 